UCTP-103/1992

\title{
A New Field Theoretic Approach to Criticality
}

\author{
P. SURANYI ${ }^{\dagger}$ \\ Department of Physics, University of Cincinnati, \\ Cincinnati, Ohio 45221-0011, USA
}

\begin{abstract}
A reorganized perturbation expansion with a propagator of soft infrared behavior is used to study the critical behavior of the mass gap. The condition of relativistic covariance fixes the form of the soft propagator. Finite approximants to the correlation critical exponent can be obtained in every order of the modified, soft perturbation expansion. Alternatively, a convergent series of exponents in large orders of the soft perturbation expansion is provided by the renormalization group in all spatial dimensions, $1 \leq D \leq 3$. The result of the $\epsilon$-expansion is recovered in the $D \rightarrow 3$ limit.
\end{abstract}

\footnotetext{
† E-mail: suranyi@ucbeh.san.uc.edu PACS numbers 11.10Ef and 64.60.Fr
} 


\section{Introduction}

The critical behavior of various statistical systems near continuous phase transitions has been the subject of intense investigations for several decades. Continuum quantum field theory turned out to be the most appropriate tool for these investigations. It connects critical phenomena with the fundamental features of renormalization and renormalization group. Nevertheless, the best numerical values for critical parameters are obtained more often then not from lattice methods: high and low temperature expansions, Monte-Carlo investigations, etc. The reason for this dichotomy is obvious. Numerical methods on lattices are convergent procedures, which, as a function of parameters (or computer time), diverge only exactly at the critical point. In contrast to that, the critical behavior in continuum field theories is investigated through the $\epsilon$-expansion of Wilson and Fisher ${ }^{[1]}$, which define only an asymptotic series for all values of the expansion parameter, $\epsilon^{[2]}$

In the current paper, we intend to introduce a new method for the investigation of critical behavior in quantum field theories. The method is based on a reorganization of the perturbation expansion series, very similar to mass renormalization, with the intent of extracting key nonperturbative features from field theories. The purpose of the modification of the propagator is to soften the infrared behavior of the propagator, thus providing a finite approximant to the correlation critical exponent in every order of the perturbation expansion. The condition of relativistic covariance fixes the form of the softened propagator up to a scale transformation. The form of the propagator is simply related to the correlation exponent, $\nu$. The series of approximants for $\nu$, obtained from successive orders of the soft perturbation expansion, is convergent, or at least has a convergent subseries. In this respect, our method is unique.

The method will be demonstrated on the example of the $\phi^{4}$. In the next section, the modified perturbation expansion will be motivated and defined. Sec. 3. will prove the existence of a soft propagator consistent with the requirement of 
correct relativistic form of the energy of single particle states. The renormalization group approach will be applied to the theory defined by the modified perturbation expansion in Sec. 4. We will prove that an alternative series of exponents can be obtained in every spatial dimension, $1 \leq D \leq 3$. Near $D=3$ the leading order approximation reproduces the one loop result of $\epsilon$-expansion. In the final section, our results will be summarized.

\section{Modified Perturbation Expansion}

The stationary state Schrödinger equation for $\phi^{4}$ field theory in $D$ spatial dimensions is given by

$$
H|\psi\rangle=\frac{1}{2} \int d^{D} x\left(\Pi^{2}(x)+m^{2} \phi(x)+\partial_{\mu} \phi(x) \partial_{\mu} \phi(x)+\frac{g}{12} \phi^{4}(x)\right)|\psi\rangle=E|\psi\rangle,
$$

The investigation of a field theory in Schrödinger representation is easiest using the representation

$$
\Pi(x)=\frac{1}{i} \frac{\delta}{\delta \phi(x)}
$$

for the canonical momentum operator. Then one can choose $|\psi\rangle$ to be a functional of the classical field $\phi^{[3]}$ This corresponds to the coordinate representation of quantum mechanics.

We will investigate the equations for the ground and first excited (single particle) states simultaneously. Our aim is to extract information concerning the critical behavior of the renormalized mass. We will denote the energy eigenvalues of the ground state and the first excited state by $E^{(0)}$ and $E^{(1)}=E(\mathbf{q})+E^{(0)}$, respectively. The first excited state of the system is a single particle state. The excitation energy of a single particle state must have the correct relativistic form

$$
E^{2}(\mathbf{q})=\mathbf{q}^{2}+m^{2}
$$

where the notation $m$ is used for the physical mass. 
As usual, we rearrange the Hamiltonian by subtracting a mass renormalization term,

$$
\delta H=\frac{\delta m^{2}}{2} \int d^{D} x \phi^{2}(x)
$$

from the kinetic part and adding it to the interaction part. Then, with an appropriately chosen $\delta m^{2}=m_{0}^{2}-m^{2}$, the perturbation expansion becomes finite for $D<3$. We define the subtraction scheme more precisely below.

The Hamiltonian perturbation expansion (or old fashioned perturbation expansion) is completely equivalent to the covariant perturbation expansion. After collecting terms in every order of the perturbation expansion, one can introduce the extra $p_{0}$ momentum components and get an expression for the squared energy function $E^{2}(\mathbf{p})$ as

$$
E^{2}(\mathbf{p})=\mathbf{p}^{2}+m^{2}+\left.\Sigma\left(p_{0}, \mathbf{p}\right)\right|_{p_{0}^{2}=\mathbf{p}^{2}+m^{2}},
$$

where $\Sigma\left(p_{0}, \mathbf{p}\right)$ is the sum of self-energy corrections. The self-energy correction is covariant, thus, it depends on $p^{2}=p_{0}^{2}+\mathbf{p}^{2}$ only, but for future reference we indicate its dependence on the energy component independently. Furthermore, if $m$ is the renormalized mass, the self-energy correction must be subtracted as $\Sigma(m, 0)=0$. Then, of course, every term of the covariant perturbation expansion of $\Sigma$, being momentum independent, vanishes for all p. The reader may ask at this point, why do we go through this elaborate procedure to define an identically zero quantity? The answer is that we will use a similar procedure later, in which the vanishing of $\Sigma$ will not be trivial at all, and at that point we will draw on the analogy to the trivial excercise above.

The information concerning the critical behavior extracted from the perturbation expansion is of a divergent nature. The root of this problem is shown clearly by the $m$ dependence of the terms of the perturbation expansion of $\delta m^{2}$. Dimensional considerations show that in $n$th order of the perturbation expansion, after 
the removal of a term diverging when the cutoff, $\Lambda \rightarrow \infty$, but independent of $m$, one is left with a term depending on the renormalized mass as

$$
\delta m^{2}=c_{n} g^{n} \mu^{n(3-D)} m^{2-(3-D) n}
$$

where $c_{n}$ is a dimensionless constant. For every $1 \leq D<3$ and for sufficiently large $n$, the power of $m$ becomes negative. On the other hand, the dependence of the 'exact' $\delta m^{2}$ on $m$ is related simply to the critical behavior

$$
\delta m^{2}=m_{c}^{2}(\Lambda)+c m^{\frac{1}{\nu}}+O\left(m^{\lambda}\right)
$$

where $\lambda>1 / \nu$ and $\nu$ is the correlation critical exponent and $m_{c}^{2}(\Lambda)$ is the critical value of the squared bare mass. Thus, the $m \rightarrow 0$ behavior of (2.6) has nothing to do with the real critical behavior.

Our thesis is that one does not obtain the correct critical behavior because some crucial non-perturbative features of the theory are overlooked by a straightforward perturbative expansion. Our aim is to incorporate some non-perturbative features into the expansion such that the critical behavior of the renormalized mass can be extracted. The procedure we intend to employ is very similar to the additive renormalization of mass.

There are examples of dramatic improvement of the convergence of perturbation series by reorganizing the perturbation series. Halliday and Suranyi were able to obtain convergent perturbation expansions for the anharmonic oscillator in quantum mechanics by an appropriate reorgnization of the series. ${ }^{[4[5]}$ Following the spirit of these works, we will alter the propagator by adding a term

$$
\delta H=\frac{1}{2} \int d^{D} p \Delta(\mathbf{p}) \phi^{\dagger}(\mathbf{p}) \phi(\mathbf{p})
$$

to the kinetic part of the Hamiltonian and subtracting it from the potential part. By this rearrangement, we are not able to make the perturbation series convergent 
for non-universal quantities like the coefficient of the term $m^{1 / \nu}$, but we are able to extract convergent approximations for the exponent $\nu$.

At this point, the form of the corrected propagator is undetermined. Using an arbitrary propagator, however, changes the perturbation series in an uncontrolled manner, thus, the series expansion for $E(\mathbf{p})$ will not have the correct relativistic form (2.6). We will show in the next section that requiring the correct relativistic form for $E^{2}(\mathbf{p})$ in every order of the perturbation expansion fixes the form of $\Delta(\mathbf{p})$, up to an irrelevant overall multiplier.

It will be convenient to use the $D+1$ dimensional, 'relativistic' form of the perturbation expansion. Then the propagator will take the form

$$
D\left(\mathbf{p}, p_{0}\right)=\frac{1}{p_{0}^{2}-\omega^{2}(\mathbf{p})+i \epsilon}
$$

where

$$
\omega^{2}(\mathbf{p})=\mathbf{p}^{2}+m^{2}+\Delta(\mathbf{p})
$$

while the interaction term will be

$$
\begin{aligned}
L_{\mathrm{int}} & =\frac{1}{2} \int d^{D+1} x\left[\frac{g}{12} \phi^{4}(x)+\delta m^{2} \phi^{2}(x)\right]-\frac{1}{2} \int d^{D+1} p \Delta(\mathbf{p})|\phi(p)|^{2} \\
& =L_{\mathrm{int}}+L_{\delta m^{2}}+L_{\Delta},
\end{aligned}
$$

where for convenience we have given $L_{\Delta}$ in momentum representation.

It is easy to see, starting from the $D$ dimensional, Hamiltonian form of the theory, that in the language of the $D+1$ dimensional 'relativistic' theory, the quantity we must calculate is

$$
E^{2}(\mathbf{q})=\omega^{2}(\mathbf{q})+\left.\Sigma\left(q_{0}, \mathbf{q}\right)\right|_{q_{0}^{2}=\omega^{2}(\mathbf{q})}
$$

In contrast to the similar expression obtained in the standard perturbation expansion, (2.5), one does not trivially obtain the correct relativistic form for the single 
particle energy. Requiring the correct relativistic form (2.6) implies the nontrivial integral equation

$$
\Delta(\mathbf{q})+\left.\Sigma\left(q_{0}, \mathbf{q}\right)\right|_{q_{0}^{2}=\mathbf{q}^{2}+m^{2}+\Delta(\mathbf{q})}=0
$$

for $\Delta(\mathbf{q})^{1}$

There is a trivial solution of $(2.13), \Delta(\mathbf{q}) \equiv 0$, reducing (2.13) to (2.5). We will prove in the next section that a nontrivial solution for $\Delta(\mathbf{q})$ exists as well such that in every order of the modified perturbaton expansion (2.13) is satisfied and the correct dispersion relation for the energy is maintained.

\section{Existence of the Modified Expansion}

The perturbative terms become bounded functions of the physical mass, $m$, unlike (2.6), only if the infrared behavior of the propagator is softened. Thus $\Delta(\mathbf{p})$ should have a leading order infrared behavior

$$
\Delta(\mathbf{p}) \simeq|\mathbf{p}|^{2 \alpha} \xi^{2-2 \alpha}
$$

where $0<\alpha<1$ and $\xi$ is a scale parameter of the dimension of a mass. Then the $\mathbf{p}$ dependence of the propagator is dominated by $\Delta(\mathbf{p})$ in the interval $I$,

$$
I: \xi>>|\mathbf{p}|>>m
$$

The euclidean propagator of the 'relativistic' perturbation theory has the form

$$
D\left(p_{0}, \mathbf{p}\right)=\frac{1}{p_{0}^{2}+\mathbf{p}^{2}+\xi^{2-2 \alpha}|\mathbf{p}|^{2 \alpha}+m^{2}+\ldots} .
$$

1 In a previous work, we obtained results for critical exponents, identical to the ones obtained in this paper, by a different interpretation of $(2.12)^{[6]}$ We will comment on that interpretation in Sec. 5 . 
In interval $I$, the propagator can be approximated by

$$
D\left(p_{0}, \mathbf{p}\right)=\frac{1}{p_{0}^{2}+|\mathbf{p}|^{2 \alpha}}
$$

where we absorbed the scale factor into momentum p (in other words, we set $\xi=1)$.

For the time being, we will omit term $L_{\Delta}$ of (2.11) in the perturbation expansion. We will prove later that its inclusion would not change our results for the critical exponents.

It will be sufficient to study (2.13) in interval $I$ to fix the form of $\Delta(\mathbf{p})$. We can argue that if we wish to extract the leading behavior of the terms of our expansion while the external momentum is in interval $I$, then it is self consistent to use propagator (3.4), instead of the complete form (3.3). That is certainly so if the loop integrals are dominated by contributions in which all internal momenta are in interval $I$ as well.

An $n$ loop contribution generated by such propagators has the behavior $|\mathbf{q}|^{a_{n}}$, where $a_{n}=2 \alpha+n(D-3 \alpha)$. This can be seen if we realize that in a $\phi^{4}$ theory there are $2 n-1$ propagators, each contributing $|\mathbf{p}|^{-2 \alpha}$, while the momentum integrations over $\mathbf{p}$ and $p_{0}$ contribute $|\mathbf{p}|^{D n}$ and $|\mathbf{p}|^{n \alpha}$, respectively ${ }^{1}$. Using similar arguments, it is easy to see that $n$ loop vertex corrections have the overall momentum dependence $|\mathbf{q}|^{b_{n}}$, where $b_{n}=n(D-3 \alpha)$. Now a necessary condition for the finiteness of self energy corrections is the finiteness of vertex corections. Those are finite only if

$$
\alpha>\alpha_{0}=\frac{D}{3}
$$

We will restrict ourselves to such values of $\alpha$ in the future.

1 Note that $p_{0}=O\left(|\mathbf{p}|^{\alpha}\right)$ in loop momenta. 
Suppose now that in a certain loop integral the loop momentum is in the region $|\mathbf{p}| \geq \mu$, but the momenta external to the loop are in $I$. Then the loop is effectively shrunk to a point vertex with at least four external legs (self energy insertions will be discussed below). If the loop contains $k$ propagators $(k \geq 2)$, then the overall behavior of the diagram has been increased by a power of $2 k \alpha-\alpha-D>0$, in view of the constraint (3.5) on $\alpha$. Then we do not obtain a leading contribution, since $|\mathbf{q}|<<\xi=1$.

If the external momentum of a self energy insertion is in $I$, but internal momenta are not, then the correction is even smaller. This can be seen by observing that self energy corrections are subtracted at $p_{0}^{2}=\mathbf{p}^{2}=0\left(m^{2}\right.$ can be neglected in $I$ ) and the largest contribution must be proportional to $p_{0}^{2}$. Then observing that $p_{0}^{2}=O\left(|\mathbf{p}|^{2 \alpha}\right)>>|\mathbf{p}|^{a_{k}}$, for all $k \geq 1$, we can see that again we obtain a non-leading contribution. Thus we have proved that if the external momentum is in interval $I$, then propagator (3.4) should be used.

In $N$ th order of the modified expansion, the infrared behavior of the diagrams is controlled by power $a_{n}=2 \alpha+n(D-3 \alpha)$. If $a_{n}>0$, diagrams are infrared convergent, even at vanishing external momentum. We can easily see that the subtraction at $q_{0}=\mathbf{q}=0$ makes the contribution finite as long as $b_{n}=n(D-3 \alpha)<$ 0 , where ultraviolet divergences will appear. Then the subtracted $n$ loop integral is finite in interval $J_{n}$

$$
J_{n}: \frac{D}{3-\frac{2}{n}}>\alpha>\frac{D}{3}
$$

In the subtraction term, we set $q_{0}=0$ and $\mathbf{q}=0$. The subtraction results in differences having large momentum behavior

$$
|\mathbf{p}-\mathbf{q}|^{2 \alpha}-|\mathbf{p}|^{2 \alpha}=|\mathbf{p}|^{2 \alpha-2}\left[\alpha|\mathbf{q}|^{2}-2 \alpha \mathbf{p} \cdot \mathbf{q}+2 \alpha(\alpha-1) \frac{(\mathbf{q} \cdot \mathbf{p})^{2}}{\mathbf{p}^{2}}+\ldots\right]
$$

where $\mathbf{p}$ is a loop momentum. Since the leading correction term, $-2 \alpha \mathbf{p} \cdot \mathbf{q}$, cancels after angular integrations over $\mathbf{p}$, the ultraviolet power behavior is reduced by two. 
The subtraction in an expression of the form $\left(p_{0}-q_{0}\right)^{2}-p_{0}^{2}=q_{0}^{2}-2 p_{0} q_{0}$ reduces the power behavior by $p_{0}^{2} \sim|\mathbf{p}|^{2 \alpha}$ only $^{1}$. In other words, the ultraviolet behavior of the subtracted self-energy correction term is controlled by the power $a_{n}-2 \alpha=b_{n}$. This is, of couurse, the same power as the one controlling the ultraviolet behavior of the vertex part. Our assertion concerning the convergence of the term of the expansion in interval $J_{n},(3.6)$, has been proved.

\subsection{Existence OF A NONTRIVIAL SOLUTiON}

Next we show that a value for $\alpha \in J_{n}$ can be found such that the leading term in (2.13) vanishes. Non-leading order terms that might appear in (2.13) and non-leading terms of $\Delta(\mathbf{q})$ will be considered later. Suppose we work in $n$ loop order of our approximation scheme. Then if $|\mathbf{q}|$ is in interval $I$, the largest order ( $n$ loop) term dominates. This is so, because the series of exponents $a_{k}$ satisfies $a_{k}>a_{k+1}$. Since $|\mathbf{q}|^{2 \alpha}<<|\mathbf{q}|^{a_{n}}$, solution (3.1) of (2.13) is possible only if the coefficient of the term $|\mathbf{q}|^{a_{n}}$ vanishes.

First, in a given order of the loop expansion all euclidean diagrams are real and have the same sign. The subtraction (mass renormalization) term of every diagram becomes infrared singular at $a_{n}=0$. Since the subtraction term is always negative, the $n$ loop term tends to $-\infty$ if $\alpha \rightarrow D /(3-2 / n)$. On the other hand, it is also easy to see that the $n$-loop contribution tends to $+\infty$ at $\alpha \rightarrow D / 3$, the point of ultraviolet divergence. The contributions are analytic functions of the variable $q_{0}^{2}$. In other words, we can write a dispersion relation for the subtracted contribution

$$
\int^{\infty} d z\left[\frac{f(z,|\mathbf{q}|)}{q_{0}^{2}+z}-\frac{f(z, 0)}{z}\right]
$$

where the positive definite discontinuity has an asymptotic behavior $f(z, \mathbf{q}) \sim$ $z^{a_{n} / 2 \alpha}$. Note that the constraint $0<a_{n}<2 \alpha$ insures the convergence of (3.8).

\footnotetext{
1 Note that $|\mathbf{p}|^{2 \alpha}>>\mathbf{p}^{2}$, if $|\mathbf{p}| \in I$.
} 
Dimensional considerations show that $|\mathbf{q}|$ can be scaled out from (3.8) to give

$$
|\mathbf{q}|^{a_{N}} \int^{\infty} d z\left[\frac{f(z,|\hat{1}|)}{q_{0}^{2} /|\mathbf{q}|^{2 \alpha}+z}-\frac{f(z, 0)}{z}\right] .
$$

Furthermore, according to our previous discussions, the leading asymptotic behavior of the $n$ loop term comes from the term $q_{0}^{2}$ in the denominator of the integrand. Thus, at large values of $z$, we can also set the momentum $\hat{1}$ equal to zero in the argument of the spectral density. Finally, according to our prescription, we have to set $q_{0}^{2}=-\mathbf{q}^{2}-m^{2}-\Delta(\mathbf{q}) \simeq-|\mathbf{q}|^{2 \alpha}$, for the Euclidean $q_{0}$. Then we obtain the following expression for the large $z$ contribution, responsible for the ultraviolet divergence

$$
|\mathbf{q}|^{a_{n}} \int^{\infty} d z f(z, 0) \frac{1}{z(z-1)} .
$$

(3.10) shows that the integral indeed tends to $+\infty$ when $\alpha \rightarrow D / 3$. We can conclude that the $n$ loop contribution to the subtracted self energy diagram has a zero at some $\alpha_{n} \in J_{n}$. At $\alpha=\alpha_{n}$, the coefficient of the leading power, $|\mathbf{q}|^{a_{n}}$, where $a_{n}=2 \alpha_{n}+n\left(D-3 \alpha_{n}\right)$, vanishes.

We turn now to the discussion of non-leading order terms in $\Delta(\mathbf{q})$. Though by setting $\alpha=\alpha_{n}$, the $n$-loop contribution vanishes, the correct dispersion relation for $E^{2}(\mathbf{q})$ requires that the coefficients of the powers $|\mathbf{q}|^{a_{n}^{k}}$ (k-loop term), where $\alpha_{n}^{k}=2 \alpha_{n}+k\left(D-3 \alpha_{n}\right), k<n$ should also vanish. For $k>0$, these powers are also smaller than $2 \alpha$ and give contributions much larger then $|\mathbf{q}|^{2 \alpha}$ in the region $|\mathbf{q}|<<1$, so they should also be cancelled in (2.13). It is easy to see, however, that a non-leading term of $\Delta(\mathbf{p})$ in the propagators of the loop diagram can cancel these contributions as well as the term $\Delta(\mathbf{q})$ appearing in (2.13). Thus $\Delta(\mathbf{p})$ should have the form

$$
\Delta(\mathbf{p})=\sum_{l=0} g^{l} c_{l}|\mathbf{p}|^{2 \alpha+l(3 \alpha-D)} .
$$

Then an expansion around the leading contribution $c_{0}|\mathbf{p}|^{2 \alpha}$ leads to correction terms to the $n$ loop contribution, which have exactly the same behavior as the 
$n-1, n-2$, etc. loop terms. An appropriate choice of the coefficients $c_{l}$ in (3.11) cancels these contributions. It is amusing to notice that in large orders of the perturbation expansion the coefficients $c_{i}$ decrease very fast with $n$, e.g. $c_{1} \sim c_{0}^{3} / n^{2}$.

Finally, let us return to the neglected term, $L_{\Delta}$, of the perturbation expansion. It is easy to show that the contributions of $L_{\Delta}$ are similar to those of $k$-loop terms, $k<n$, of the perturbation expansion. Indeed, if we work in $n$ loop order, an insertion $\Delta(\mathbf{p})$ on a propagator would substitute a four point vertex, lowering the power of $g$ by one, and would substitute one of the propagators $D\left(q_{0}, \mathbf{p}\right)$ by $D\left(q_{0}, \mathbf{p}\right) \Delta(\mathbf{p}) D\left(q_{0}, \mathbf{p}\right)=O\left(D\left(p_{0}, \mathbf{p}\right)\right)$. In other words, we obtain a contribution with the same power of $g$ and the same low momentum behavior as the $n-1$ loop term. We know, however, that the $n$ loop term provides the dominant power of the expansion, thus the equation determining $\alpha$ is not affected by the term $L_{\Delta}$ of $L^{\prime}$, defined in (2.11). These non-leading terms can also be cancelled in (2.13) successively, by non-leading terms of the asymptotic expansion of $\Delta(\mathbf{p})$.

\subsection{CRitical Exponent}

The correlation critical exponent $\nu$ relates the behavior of the unrenormalized mass and the renormalized mass

$$
m_{0}^{2}=m^{2}+\delta m^{2}=m^{2}+f(\Lambda)+c m^{\frac{1}{\nu}}
$$

where $f(\Lambda)$ is the cutoff dependent critical point for the square of the bare mass and $c$ is a constant. The exponent $\alpha_{n}$ has a direct connection with the $n$th order approximant of $\nu$. One is able to extract $\nu$ if one investigates the behavior of the terms of the modified expansion for $\delta m^{2}$ in the transition region, $|\mathbf{q}| \simeq m$. In that region, the mass term cannot be neglected and we have to use the form $p_{0}^{2}+|\mathbf{p}|^{2 \alpha}+m^{2}$ for the propagator. 
Due to the infrared convergence of the integrals in interval (3.6) we can separate terms of the form $c_{k} g^{k} \Lambda^{\alpha_{n}^{k}}$ from the mass renormalization constant, such that the remainder is a finite function $m$. Then we can scale out the mass, $m$, from the terms of the perturbation expansion with the simultaneous substitutions $\mathbf{p} \rightarrow m^{\frac{1}{\alpha}} \mathbf{p}$ and $p_{0} \rightarrow m p_{0}$. Using (3.12), we obtain the following expression for $\nu_{n}$, the estimate for the critical exponent $\nu$ in $n$-loop approximation,

$$
\frac{1}{\nu_{n}}=2-n\left(3-\frac{D}{\alpha_{n}}\right)
$$

Equation (3.13) shows that the dominant contribution (smallest power of $m$ ) comes from the leading order, $n$-loop term, since for $k<n$ we have $2-k(3-$ $\left.D / \alpha_{n}\right)>2-n\left(3-D / \alpha_{n}\right)$, provided $\alpha \in J_{n}$ of (3.6). Furthermore, it also follows from (3.6) that the value of $\nu$ is between $\frac{1}{2}$ and $\infty$, as it indeed should be.

The natural method for finding approximants to $\nu$ would be to find values for $\alpha_{n}$ at as high $n$ as possible. Indeed, in a previous paper we found the critical exponent for $D=1$ and $D=2$ in the two-loop approximation, $\nu^{(1)}=1.013$, $\nu^{(2)}=0.713$, in reasonable agreement with known values. ${ }^{[6]}$

There is a puzzling question concerning our approximation scheme. Since $\alpha_{n}$ is obtained from $n$-loop diagrams only, completely different from the $(n-1)$-loop diagrams used for obtaining $\alpha_{n-1}$, it is hard to see the relation between subsequent approximants to $\nu, \nu_{n}$ and $\nu_{n-1}$. It could happen that $\nu_{n}$, as a function of $n$, oscillates between $\frac{1}{2}$ and $\infty$, instead of being convergent. A bounded infinite series always has at least one convergent subseries, but it may have several that converge to different limits. As is shown by (3.13), a smooth limit to the correct value of $\nu$ is possible only if we can prove that in large orders of the loop expansion the equation determining $\alpha$ becomes a function of $\rho=n(3-D / \alpha)$ only, thus making the existence of a nontrivial limit $\rho \rightarrow \rho_{c}$ for $n \rightarrow \infty$ possible. Then, of course, $\nu=\frac{1}{2-\rho_{c}}$. 
The simplest method for the investigation of large orders of the perturbation expansion is the renormalization group. Observe that the modified loop expansion is equivalent to a perturbation expansion in an euclidean field theory with action ${ }^{1}$

$$
\begin{aligned}
S(\phi) & =\frac{1}{2} \int d p_{0} d^{D} p\left[p_{0}^{2}+|\mathbf{p}|^{2 \alpha}\right] \phi(p) \phi(-p) \\
& +\frac{1}{4 !} \int d x_{0} d^{D} x \phi(x)^{4}+\frac{\delta m^{2}}{2} \int d p_{0} d^{D} p \phi(p) \phi(-p),
\end{aligned}
$$

Similar field theories have been investigated in the past. The closest example is that of Fisher and Grinstein ${ }^{[7]}$ who found that certain superconducting systems may be described by Hamiltonians similar to (3.14). They, however use a 'double $\epsilon$ expansion' in the variables $\epsilon=3-D$ and $\epsilon^{\prime}=1-\alpha$. Our intention is to investigate field theory (3.14), at fixed $D$ near the critical point $\alpha=D / 3$. In other words, we wish to perform an expansion in $\delta=3 \alpha-D$.

We will show in the next section that the Callan-Symanzik equation may be used to find the coefficients of terms, singular in $\delta$, in large order contributions to Green's function. This is quite analogous to the calculation of leading logarithms in perturbation theory. We will also find that up to a multiplier, independent of the momentum, the large order contribution to the self energy correction becomes a function of the variable $\rho=3 n \delta / D$. Higher and higher orders of the loop expansion provide a power series of higher and higher order in variable $\rho$. Finding the zero of the self energy correction as a function of $\rho$ provides an alternative method for the determination of $\nu$. One outstanding feature of the power series expansion in $\rho$ is that in contrast to the $\epsilon$-expansion, it has a finite radius of convergence.

1 For the sake of simplicity, we mix coordinate space and momentum space representations for $\phi$. The action could be written in either of these representations without any difficulty. 


\section{Renormalization Group}

\subsection{CAllan-SymanziK EQUATions}

The renormalization of the field theory, defined by action (3.14) requires wave function and coupling constant renormalizations, besides the additive mass renormalization we discussed earlier. The renormalized and bare amputed Green's functions have the following relation with each other

$$
\Gamma^{(4)}\left(p_{1}, p_{2}, p_{3}, p_{4}, g, \mu\right)=Z^{-2}(g, \delta) \Gamma_{0}^{(4)}\left(p_{1}, p_{2}, p_{3}, p_{4}, g_{0}, \delta\right),
$$

and

$$
\Gamma^{(2)}(p, g, \mu)=Z^{-1}(g, \delta) \Gamma_{0}^{(2)}\left(p, g_{0}, \delta\right),
$$

where $\delta=3 \alpha-D$ and $\mu$ is the renormalization scale, defined through the relation of the renormalized and the unrenormalized charges

$$
g_{0}=g \mu^{\delta} Z_{g}(g, \delta)
$$

$p_{i}$ denote both the spatial, $D$ dimensional part of the momenta, and their timelike part as well. $\Gamma^{(2)}$ is the inverse propagator, the main subject of our investigation.

The derivatives of the left hand sides of (4.1) and (4.3) with respect to $1 / \delta$ should vanish as $\delta^{2}$, when $\delta \rightarrow 0$, because their first nonvanishing $\delta$-dependent terms are proportional to $\delta$. Thus the unrenormalized Green's functions satisfy the following equations

$$
\left[\frac{\partial}{\partial 1 / \delta}+\beta(g, \delta) \frac{\partial}{\partial g}-2 \gamma(g, \delta)\right] \Gamma_{0}^{(4)}\left(p_{1}, \ldots p_{4}, g, \delta\right)=O\left(\delta^{2}\right),
$$

and

$$
\left[\frac{\partial}{\partial 1 / \delta}+\beta(g, \delta) \frac{\partial}{\partial g}-\gamma(g, \delta)\right] \Gamma_{0}^{(2)}(p, g, \delta)=O\left(\delta^{2}\right),
$$

where we dropped the subscript 0 of the coupling constant. Strictly speaking, it is not exactly $\Gamma^{(2)}$ that satisfies (4.5), as it will soon become obvious. The relation 
between the standard cutoff dependent form of the Callan-Symanzik equations and the above forms is given by the two possible limits

$$
\ln (\Lambda / \mu) \leftarrow \frac{1-(\Lambda / \mu)^{-\delta}}{\delta} \rightarrow \frac{1}{\delta}
$$

While the cutoff scheme is obtained if one takes the limit $\delta \rightarrow 0$ first, the dimensional regularization scheme is obtained if the limit $\Lambda \rightarrow \infty$ is taken first. The beta and gamma functions can be determined from (4.4) and (4.5), taken at specific momentum values. In the $\delta \rightarrow 0$ limit they coincide with the standard definitions.

The renormalization group functions have the following perturbation expansion

$$
\beta(g)=\beta_{2} g^{2}+\beta_{3} g^{3}+\ldots
$$

and

$$
\gamma(g)=\gamma_{2} g^{2}+\gamma_{3} g^{3}+\ldots
$$

\subsection{Calculation of Self energy CORRections in large orders of} THE SOFT PERTURBATION EXPANSION

The two point amplitude can be written in the form

$$
\Gamma_{0}^{(2)}\left(p_{0}, \mathbf{p}, g, \delta\right)=p_{0}^{2}+|\mathbf{p}|^{2 \alpha}+p_{0}^{2} \Xi\left(p_{0}, \mathbf{p}, g, \delta\right)
$$

where we explicitly indicated the separate dependence on $p_{0}$ and $\mathbf{p}$. The important property of $\Gamma^{(2)}$ is that the coefficient of $|\mathbf{p}|^{2 \alpha}$ does not get renormalized. When the subtraction at zero momentum is performed in the loop integrals, the leading terms in the difference are of the form $\left(p_{0}-q_{0}\right)^{2}+\left(p_{0}+q_{0}\right)^{2}-2 q_{0}^{2}=p_{0}^{2}$ and $(\mathbf{p}-\mathbf{q})^{2 \alpha}+(\mathbf{p}+\mathbf{q})^{2 \alpha}-2 \mathbf{q}^{2 \alpha} \sim \mathbf{p}^{2}<<p_{0}^{2}$, if $\mathbf{p}<<\xi$. That of course shows that 
indeed, the coefficient of $\mathbf{p}^{2 \alpha}$ does not get renormalized, and we should drop the second term from the right hand side of (4.9), when we substitute into (4.2) or $(4.5)$.

We expand the function $\Xi$ in $g$ as follows

$$
\Xi\left(p_{0}, \mathbf{p}, g, \delta\right)=\sum_{n} \frac{\left(-\beta_{2} g\right)^{n}}{\delta^{n-1}} Q_{n}(\delta, p),
$$

where the function $Q$ has the series expansion

$$
Q_{n}(\delta, p)=\sum_{m=0}^{n-1} \delta^{m} q_{n}^{m}(p)
$$

The leading coefficient $q_{n}^{0}(p)=1$, by the normalization we chose for $Q_{n}$, while $q_{n}^{n-1}(p)$ is the finite part of the $n$ loop amplitude, divided by $\left(-\beta_{2}\right)^{n}$, in the minimal subtraction scheme.

Callan-Symanzik equation (4.5) and (4.10) imply the following equation for $Q_{n}$

$$
\begin{aligned}
0 & =(n-1)\left[Q_{n}-Q_{n-1}\right]-\delta \frac{\partial Q_{n}}{\partial \delta} \\
& +\frac{\beta_{3}}{\beta_{2}^{2}}(n-2) \delta Q_{n-2}-\frac{\beta_{4}}{\beta_{2}^{3}}(n-3) Q_{n-3}+\ldots \\
& -\gamma_{2} \delta Q_{n-2}+\gamma_{3} \delta^{2} Q_{n-3}-\ldots
\end{aligned}
$$

Now $Q_{n}$ is a slowly varying function of $n$. Then, in leading order of $n$ and fixed $x=\delta n$ one obtains the following differential equaton for $Q_{n}$

$$
0=n \frac{\partial Q_{n}}{\partial n}-\delta \frac{\partial Q_{n}}{\partial \delta}+\frac{\beta_{3}}{\beta_{2}^{2}} x Q_{n}
$$

Solving (4.13) one obtains the solution of (4.12) as

$$
Q_{n}=F(x) \exp \left\{\frac{\beta_{3}}{\beta_{2}^{2}} x \log n\right\}[1+O(1 / n)]
$$

where $F(x)$ is to be determined by the inital conditions. 
Note that all the momentum dependence is concentrated in $F(x)$. Nevertheless, since $F(x)$ is dimensionless, it becomes independent of the momentum components after the substitution $p_{0}=|\mathbf{p}|^{\alpha}$. The zero of $F(x)$, as a function of $x$ determines the value of $\alpha_{n}$. As $x=D \rho / 3$, it provides solutions at fixed $\rho$, as required by the existence of a nontrivial asymptotic value for the critical exponent.

The initial conditions fix the form of $F(x)$. In fact iterating the expanded form (4.12), with $\beta_{3}=\beta_{4}=\ldots=\gamma_{2}=\gamma_{3}=\ldots=0$ we obtain

$$
F(x)=\sum_{m=0}^{n-1} \frac{x^{m}}{m !} q_{m+1}^{m} .
$$

As we mentioned before, the coefficient $q_{m+1}^{m}$ is the finite part of the $m$ loop term, divided by $\left(-\beta_{2}\right)^{m}$, in the minimal subtraction scheme.

It is well known that in a $\phi^{4}$ field theory the asymptotic $m$ dependence of $m$ loop contributions to Green's functions $\Gamma_{n} \sim(-1)^{m} m ! a^{-m} m^{k} b .^{[2]}$ Consequently, expansion (4.15) of $F(x)$ has a finite radius of convergence, $a$. The improved convergence of series (4.15) compared to that of the $\epsilon$-expansion is due to the extracted multipliers $n^{m}$, contained in the power $x^{m}$.

The finite radius of convergence, and the fact that the singularities of the expansion series (4.15) are at $x<0$ (the series is alternating) allows a conformal transformation of the variable $x$ such that all values $0<\rho<2$ lie in the domain of convergence.

Unfortunately, due to the complicated form of the propagator of the effective theory we have not been able to determine parameter $a$, controlling the radius of convergence yet. We know, however that $F(x)$ must have a zero between $0<\rho<2$. 


\subsection{CRITICAL EXPONENTS In FiRSt APPROXimation}

The leading order term of integral equation (2.13) vanishes if $\alpha$ is the root of the subtracted $n$-loop contribution. As we have seen it earlier, at large $n$ this is equivalent to finding the root of the $n$ loop contribution as a function of $x=$ $n(3 \alpha-D) \equiv n \delta$. Then $\nu=1 /(2-3 x / D)$. Since the $n$-loop contribution is proportional to $F(x)$, we have to find a root of $F(x)$. Then a series of critical exponents, $\nu_{k}$, alternative to the series obtained in Sec. 3, can be obtained by approximating $F(x)$ by its terms up to $x^{k}$.

In particular, for $k=1$ we obtain $x_{1}=-1 / q_{2}^{1}$, where $q_{2}^{1}$ is the ratio of the minimally subtracted finite term and of the pole term of the subtracted two loop self-energy diagram. Let us determine $q_{2}^{1}$. It is simpler to use old- fashioned perturbation theory (the amplitude integrated over timelike momentum components). The subtracted two loop amplitude, up to irrelevant multipliers, is

$$
\begin{aligned}
\Gamma^{(2)} & =\int \frac{d^{D} p_{1}}{\mathbf{p}_{1}^{\alpha}} \frac{d^{D} p_{2}}{\mathbf{p}_{2}^{\alpha}}\left\{\frac{1}{\left[\mathbf{p}_{1}^{\alpha}+\mathbf{p}_{2}^{\alpha}+\left(\hat{\mathbf{1}}-\mathbf{p}_{1}-\mathbf{p}_{2}\right)^{\alpha}\right]^{2}-1}\right. \\
& \left.-\frac{1}{\left[\mathbf{p}_{1}^{\alpha}+\mathbf{p}_{2}^{\alpha}+\left(\mathbf{p}_{1}+\mathbf{p}_{2}\right)^{\alpha}\right]^{2}}\right\} .
\end{aligned}
$$

In (4.16), as required, we substituted $q_{0}^{2}=-\mathbf{q}^{2 \alpha}$ for the components of the external momentum. Furthermore, we factored $\mathbf{q}^{2 D-4 \alpha}$ out. $\hat{\mathbf{1}}$ is a unit vector.

It is comparatively easy to separate the pole term from (4.16), in variable $\delta$. We obtain

$$
\Gamma_{\text {pole }}^{(2)}=\frac{\Omega_{D}}{\delta} \int_{0}^{1} d \xi \int d \Omega_{D} \frac{\xi^{2 D / 3-1}}{\left[1+\xi^{D / 3}+\left(1+\xi^{2}+2 \xi \cos \theta\right)^{D / 6}\right]^{4}}
$$


The finite part of (4.16), taken at $\alpha=D / 3$ is

$$
\begin{aligned}
\Gamma_{\text {finite }}^{(2)} & =\int \frac{d^{D} p_{1}}{\mathbf{p}_{1}^{D / 3}} \frac{d^{D} p_{2}}{\mathbf{p}_{2}^{D / 3}}\left\{\frac{1}{\left[\mathbf{p}_{1}^{D / 3}+\mathbf{p}_{2}^{D / 3}+\left(\hat{\mathbf{1}}-\mathbf{p}_{1}-\mathbf{p}_{2}\right)^{D / 3}\right]^{2}-1}\right. \\
& \left.-\frac{1}{\left[\mathbf{p}_{1}^{D / 3}+\mathbf{p}_{2}^{D / 3}+\left(\mathbf{p}_{1}+\mathbf{p}_{2}\right)^{D / 3}\right]^{2}}-\frac{\theta\left(\left|\mathbf{p}_{1}\right|-1\right) \theta\left(\left|\mathbf{p}_{2}\right|-1\right)}{\left[\mathbf{p}_{1}^{D / 3}+\mathbf{p}_{2}^{D / 3}+\left(\mathbf{p}_{1}+\mathbf{p}_{2}\right)^{D / 3}\right]^{4}}\right\} \\
& +\frac{\Omega_{D}}{3} \int_{0}^{1} d \xi \int d \Omega_{D} \xi^{2 D / 3-1}\left\{\frac{5 x^{2 D / 3-1} \ln x}{\left[1+\xi^{D / 3}+\left(1+\xi^{2}+2 \xi \cos \theta\right)^{D / 6}\right]^{4}}\right. \\
& -\frac{4 x^{D / 3} \ln x+2 \ln \left(1+x^{2}+2 x \cos \theta\right)\left(1+x^{2}+2 x \cos \theta\right)^{D / 6}}{\left[1+\xi^{D / 3}+\left(1+\xi^{2}+2 \xi \cos \theta\right)^{D / 6}\right]^{5}} .
\end{aligned}
$$

We have evaluated the ratio $x=-\Gamma_{\text {pole }}^{(2)} / \Gamma_{\text {finite }}^{(2)}$ for $D=2$. We obtained $x=.402$, resulting in the prediction for the critical exponent, $\nu=.717$, very similar to the one obtained by our first method. Furthermore we examined the limit $D \rightarrow 3$ as well. The first term of $\Gamma_{\text {finite }}^{(2)}$ develops a pole at $D=3$. Using the standard notation $\epsilon=3-D$, one obtains $\Gamma_{\text {pole }}^{(2)} / \Gamma_{\text {finite }}^{(2)}=-\epsilon / 3$, and $\nu=1 /(2-\epsilon / 3)$, in agreement with the leading order result from $\epsilon$ - expansion ${ }^{[8]}$.

\section{Summary}

A modified perturbation expansion was introduced in this paper. The expansion has the virtue of providing a potentially convergent series of approximants for the critical exponent $\nu$. The subsequent approximations are determined by subsequent orders of a modified perturbation expansion, in which the infrared behavior of the propagator is softened. The condition of relativistic invariance fixes the propagator uniquely.

The results of the first part of this paper are identical to those of a previous work of ours, ${ }^{[6]}$ in which we arrived at the approximants to the critical exponent taking a different route. In that paper we obtained a slightly different form of 
$(2.12)$

$$
E^{2}(\mathbf{q})=\mathbf{q}^{2}+m^{2}+\left.\Sigma\left(q_{0}, \mathbf{q}\right)\right|_{q_{0}^{2}=E^{2}(\mathbf{q})}
$$

which was interpreted as an integral equation for $E^{2}(\mathbf{q})$. Then a solution of nonrelativistic infrared behavior, $E^{2}(\mathbf{q}) \sim c \mathbf{q}^{\alpha}$, was obtained, where $\alpha$ coincides with the value of $\alpha$ obtained in the current paper. In Ref.[6] it was assumed that the self-consistency of (5.1) requires the vanishing of coefficient $c$ in infinite order.

The results of the second half of our paper are even more interesting. The modified perturbation theory defines an effective field theory in the infrared domain. Large orders of that effective field theory can be investigated through the renormalization group. The k-loop approximation to the solution of the renormalization group equation was shown to provide a series of finite radius of convergence, the zero of which also provides an approximation to the correlation exponent.

The prescription, we provided for the determination of the correlation exponent in $n$ loop order uses the $n$ loop terms alone. This is the very circumstance which makes the universality of the critical exponent (its independence from the coupling constant) possible. In the $\epsilon$-expansion approach the dependence on the coupling constant is eliminated by the limit $g \rightarrow g_{c}(\epsilon)$, where $g_{c}(\epsilon)$ is the fixed point of the renormalization group transformation. Here we approach the critical surface at an arbitrary value of the coupling constant, by adjusting the bare mass. Thus, $\nu$ can be independent of $g$ only if in every order it is determined by the highest loop contribution alone.

Finally, we discuss the extension of the results of this paper to the $O(N)$ model. The only difference a calculation in the $O(N)$ model would make is that the contribution of individual diagrams would be multiplied by an $N$ dependent multiplier. There is only one diagram contributing to the equation determining $\alpha_{n}$ in two and three loop order (Note that we have chosen the subtraction scheme in which momentum independent insertions on propagators cancel). Thus only in 
the four or higher loop orders (where there are four or more topologically different diagrams) would we get a $N$-dependent $\nu$. This, is certainly not a fatal flow, since the dependence of $\nu$ on $N$ is weak. As far as the $N$ dependence of the critical exponent is concerned the situation is similar in the renormalization group approach. This is a little more surprising, because for $N=1$ (but not for other $N$ ) at $D \simeq 3$ the result of the $\epsilon$-expansion is reproduced in the two loop approximation.

The author is indebted to L.C.R. Wijewardhana, for discussions, to Beth Basista, for the critical reading of the manuscript, and to Michael Ma, for calling his attention to Ref.[7]. This work has been supported in part by the US Department of Energy, through Grant DE-FG02-84ER40153. The support of the Ohio Supercomputer Center for computing time and of the Research Council of the University of Cincinnati for computer hardware is gratefully acknowledged.

\section{REFERENCES}

1. K.G.Wilson and M.E.Fisher, Phys. Rev. Lett 28 (1972), 240;

2. E. Brezin, J-C. Le Guillou, and J. Zinn-Justin, Phys. Rev. D15 (1977), 1544;

3. R. Jackiw: "Functional Representation for Quantized Fields", in Proceedings of the 1st Asia Pacific Workshop on High Energy Physics, Conformal Field Theory, Anomalies and Superstrings, ed. B.E. Baaquie et al., World Scientific, Singapore, 1988;

4. I.G. Halliday and P. Suranyi, Phys. Lett. 85B (1979), 2134;

5. I.G. Halliday and P. Suranyi, Phys. Rev. D21 (1980), 1529;

6. P. Suranyi, preprint UCTP 106/91, to be published;

7. M. P. Fisher and G. Grinstein, Phys. Rev. Lett. 60 (1988), 208;

8. K.G. Wilson and J. Kogut, Phys. Rep. C12 (1974), 75. 